\title{
Steady-State and Transient Conductivity of Colloidal Solutions of Gold Nanobeads
}

\author{
Enrique Valera, Ángel Rodríguez, Member, IEEE, and Luis M. Castañer, Senior Member, IEEE
}

\begin{abstract}
Steady-state and transient conductance measurements of gold nanobeads solutions deposited on top of interdigitated electrodes have been performed. It is shown that the application of an electric field of moderate value between electrodes during the drying process of the droplet makes the resulting steady-state conductance value to increase significantly. The dynamics of the gold nanobeads in the solution has been studied by means of transient current measurements during the drying process and the effects correlated to the changes in the morphology of the association of the gold nanobeads when they reach the substrate. It is seen that the application of the electric field foster the formation of gold beads monolayers, chains, and dendritic associations which, in combination with the humidity conditions of the sample surface, are believed to be the reasons for the conductance increase.
\end{abstract}

Index Terms-Gold nanobeads, interdigital transducers, nanoparticles conduction.

\section{INTRODUCTION}

$\mathbf{H}$ ANDLING of particles at the micro-nano scale is an important tool to provide means of implementation of protocols and devices aimed at the detection of biomolecules or cells. In particular, gold nanobeads are currently used in electrical [1] or optical biodetection [2]. Besides biomedical applications, the dynamics of conductive nanoparticles needs to be understood for electronic applications such as lithographic patterning [3], selective deposition [4], nanoprinters [5], self-organization [6], [7], single-electron transistors [8], [9], nanoparticle dense films [10], or other nanoscale devices that apply the electrostatic trapping of single nanoparticles [11], [12].

One of the simplest ways to detect changes, and hence ascertain the presence of a given reaction, is the measurement of changes in the electrical impedance of interdigitated microelectrodes, and in particular the changes in the conductivity.

Although there are large number of devices and experiments using a combination of ID electrodes and gold particles, they usually refer to steady-state measurements and morphological observations once equilibrium has been reached in the sample. Some of the observations indicate that ordered gold colloid

Manuscript received March 31, 2006; revised May 23, 2007. This work was supported in part by the Ministry of Science and Technology under Contract TEC2004-06854-C03-03/MIC) and in part by the European Community under Project IST-2003-508774. The review of this paper was arranged by Associate Editor A. Bose.

The authors are with the Micro and Nano Technologies group (MNTg). Departament d'Enginyeria Electrònica. Universitat Politècnica de Catalunya, 08034-Barcelona Spain (e-mail: valera@eel.upc.edu; arm@eel.upc.edu; castaner@eel.upc.edu).

Digital Object Identifier 10.1109/TNANO.2007.902099

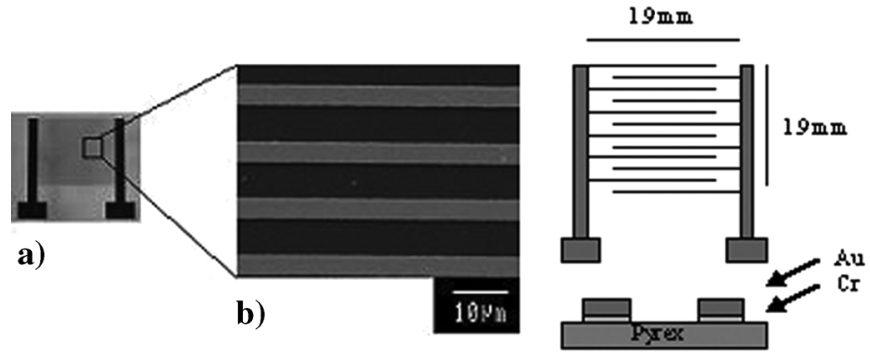

c)

Fig. 1. Interdigitated electrodes. (a) Optical image. (b) SEM image. (c) Schematic.

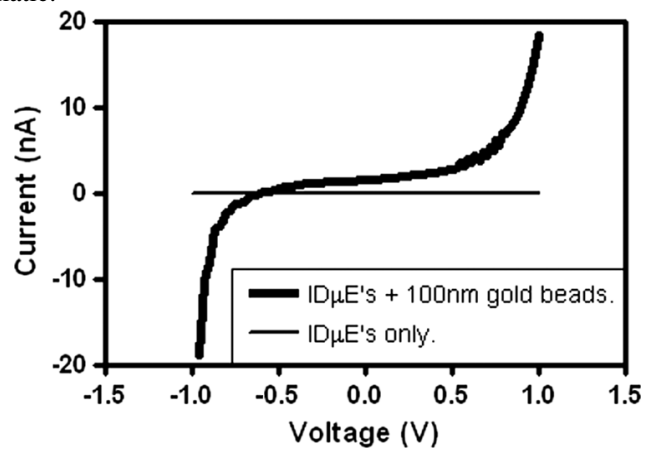

Fig. 2. Electrical $I-V$ curves of: (a) ID $\mu$ Es after $100 \mathrm{~nm}$ gold beads deposition (thick curve) and (b) ID $\mu$ Es prior to the gold beads deposition (thin curve).

lattices and monolayers can be formed [13], [14] using electrophoretic forces, gold particles fractal structures can also develop by means of chemical discharge [15], [16] and gold particles chains [17] can also be found in the literature.

The purpose of this work is to contribute to the knowledge of gold particle dynamics in colloidal solutions, by means of steady-state and transient conductance measurements. In fact the measurement of transient currents developing through the ID electrodes allows to monitor the changes in the conductivity of the solution and hence the resulting morphological association of gold beads and the $I-V$ stationary characteristics once the transient has elapsed can be related to the dynamics of the particles. In order to assess whether the electrical bias of the ID electrodes while the solution is still wet plays some role in the aggregation of the beads, an electric field has been applied just after the solution enters in contact with the IDs and the transient $I(t)$ characteristics are measured until the drop is totally dry. Steady-state $I(V)$ curves are then taken and morphological microscopy observations are also performed.

\section{EXPERIMENTAl Procedure AND Steady-State CONDUCTANCE MEASUREMENTS}

Thin $\mathrm{Au} / \mathrm{Cr}$ interdigitated microelectrodes (ID $\mu \mathrm{Es}$ ) have been patterned on a Pyrex glass substrate with a bar shape, $3.85 \mu \mathrm{m}$ 


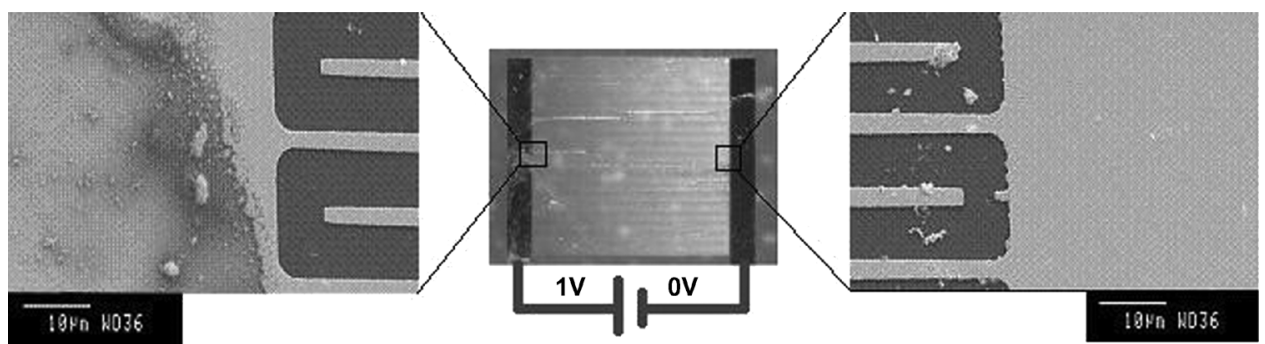

Fig. 3. Resulting distribution of gold nanobeads after bias polarization.

thick with electrode gap of $6.8 \mu \mathrm{m}$. Fig. 1 shows an optical microscope image of one of the patterns along with an enlarged SEM picture of a detail of the fingers and gaps and the schematic of the ID $\mu$ Es used.

Two different aqueous solutions have been used, one of $5.6 \times$ $10^{9}$ particle $/ \mathrm{mL}$ concentration of $100 \mathrm{~nm}$ diameter gold beads and the second of $9 \times 10^{10}$ particle $/ \mathrm{mL}$ concentration of $40 \mathrm{~nm}$ diameter gold beads. Both solutions are from British Biocell International and the gold beads have a negative charge as a means to keep the beads in suspension as they repel each other, making the colloidal solution very stable against aggregation. A micropipette was used to pour different amounts of microdroplets of the solution, between $5 \mu \mathrm{L}$ to $50 \mu \mathrm{L}$, on top of the microelectrode array. In all our experiments, drying of the drop was helped using a $100 \mathrm{~W}$ halogen lamp for about 5-35 min typically depending on the solution volume. The droplet covered totally or partially the array.

As a baseline procedure a $50 \mu \mathrm{L}$ of $100 \mathrm{~nm}$ gold solution droplet was deposited on top of the ID device and then dried using the halogen lamp.

Conductivity was measured using a HP 4145 B Equipment by means of $I-V$ curves from $-1 \mathrm{~V}$ to $+1 \mathrm{~V}$ sweep bias. The results of the measurement after the droplet was deposited and dried are compared in Fig. 2 with the same measurement of the ID prior to the colloidal solution was deposited.

As can be seen there is a large difference in the current even at moderate bias voltage values, for example at $+1 \mathrm{~V}$, the current is around $20 \mathrm{pA}$ prior to the gold solution deposition and evaporation, whereas it is in the range of $18 \mathrm{nA}$ after for the same bias. This means an increase of three orders of magnitude. Similar differences were also observed by Malaquin [1] although our current values are much larger. In this case the distribution of gold nanoparticles is quite random in the area between the electrodes.

Despite the huge conductance difference prior and after deposition, the current level is still quite low, and in order to further increase the sensitivity, we have carried out a different experiment and compared to our baseline measurements described above.

The main idea behind this experiment is to observe the effects (electrical and morphologic) of applying a bias voltage between the ID electrodes after the solution was deposited and during all the drying of the droplet. This means to observe the effects that the application of an electric field may have in the resulting conductance after the droplet is let to totally dry.

A $1 \mathrm{~V} \mathrm{dc}$ bias voltage was applied between the electrodes at the same time as the halogen lamp was ON. The process lasted

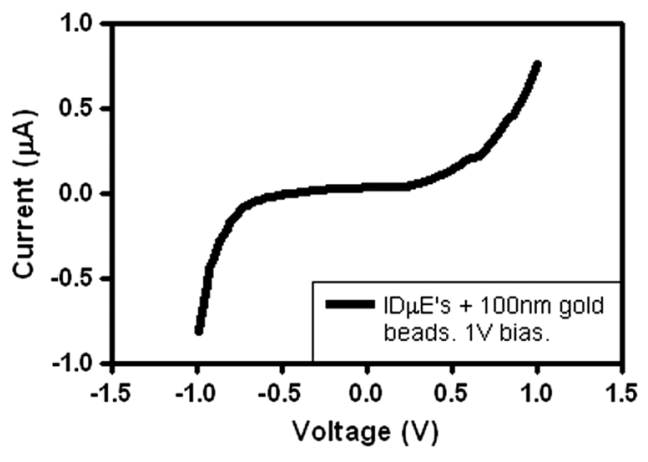

Fig. 4. $I-V$ curve for the device shown in Fig. 3 .

5-35 min typically, depending on the solution volume used. At the end of the $50 \mu \mathrm{L}$ of $100 \mathrm{~nm}$ gold solution drying process, the distribution of nanobeads of gold resulted as shown in Fig. 3.

As can be seen in Fig. 3, the positive electrode PAD (left hand side of the picture) was almost covered by gold nanobeads, whereas the negative electrode was almost uncovered as revealed by the SEM images. Additionally, in the optical image can be seen an important amount of gold nanobeads deposited on the area close to the positive main electrode PAD in comparison with the area close to the negative main electrode PAD

Conductance measurements performed after the solution was dried, with application of bias voltage during drying of the sample, are shown in Fig. 4. This $I-V$ curve is compared to the same $I-V$ curve of our baseline process, without application of bias, of Fig. 2. As can be seen a significant increase in the value of the electrical current at moderate voltage is observed, namely the value of the electrical current at $+1 \mathrm{~V}$ bias polarization is $0.8 \mu \mathrm{A}$, which is about 50 times the value given by our baseline result for the same bias voltage.

To explain those differences, morphological observations have also been done as shown in Figs. 5 and 6.

Fig. 5(a) shows a SEM image of a consistent monolayer between electrodes and Fig. 5(b) shows the detail of the structure of this monolayer.

As can be seen in Fig. 5(a) and (b), gold nanobeads monolayers have been observed in between the electrodes and also dendrite associations as shown in Fig. 6(a) and (b). Those formations have been found in several places of the surface of the device.

Such structures as monolayers, lattices, dendrites, or fractals of citrate ion-stabilized gold nanobeads had been previously reported [13]-[16] after different procedures such as electrophoresis [13], [14] purely chemical [15], [16], or by means 

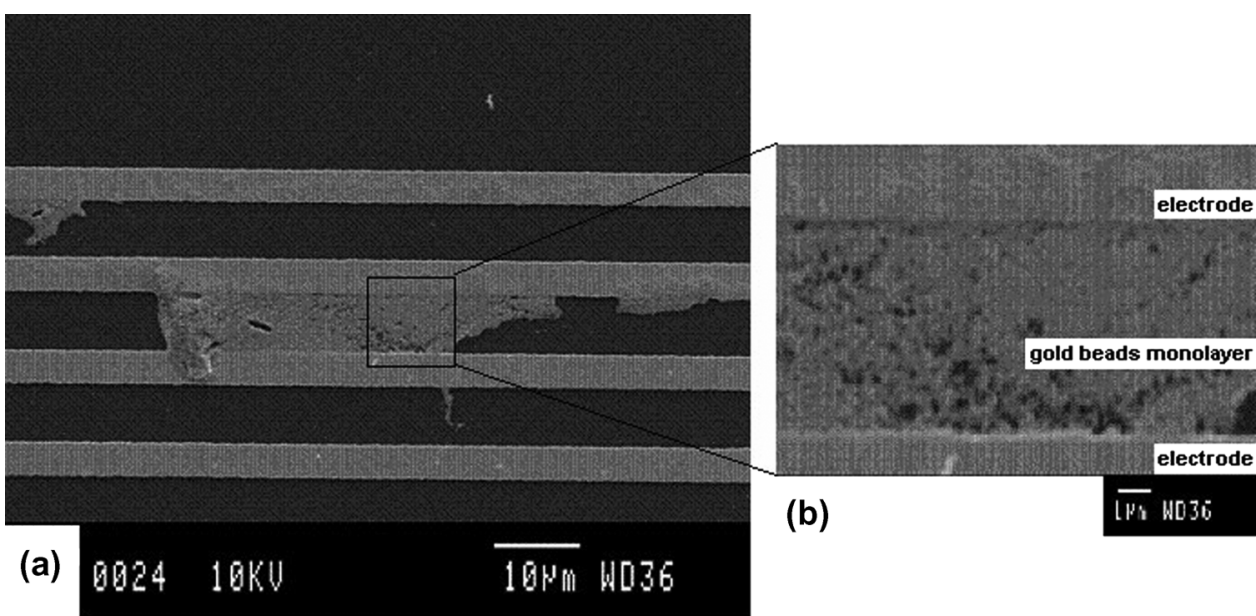

Fig. 5. Monolayer between electrodes. (a) SEM image. (b) Detail of the structure.

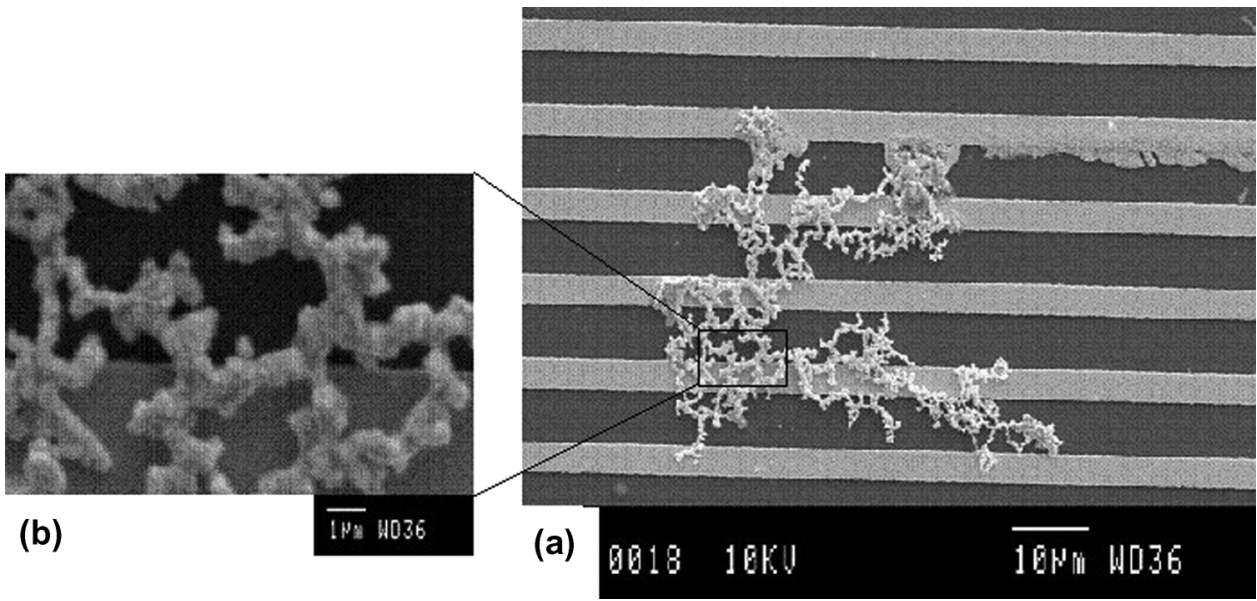

Fig. 6. Dendrite structure. (a) SEM image. (b) Detail of the chains of nanobeads.

of a prealigned functional polymer as a chemical template for the colloidal particles [17].

\section{TRANSIENT CONDUCtANCE MEASUREMENTS}

From the steady-state conductance measurements and associated morphological observations of the gold nanobeads aggregation we concluded that the application of an electric field during the drying process was responsible for the monolayer and dendrite structures formations as these were not observed in the samples where no electric field was applied. In order to better understand the gold nanobeads dynamics during the drying process, transient measurements $I(t)$ of the electrical current developing through the electrodes were performed. Transient conductance was measured using an Agilent $4156 \mathrm{C}$ Equipment. An example of the transient is shown in Fig. 7.

$I(t)$ curves for two different applied voltages are shown in Fig. 7. Apparently no changes are observed for the case where the electric field is small (namely $0.1 \mathrm{~V}$ of applied voltage). However when the applied voltage is set to $1 \mathrm{~V}$, the current transient has significant value and three main phases can be observed.

The current is initially zero prior to the solution droplet is deposited on top of the electrodes. Suddenly, in Phase I, the current

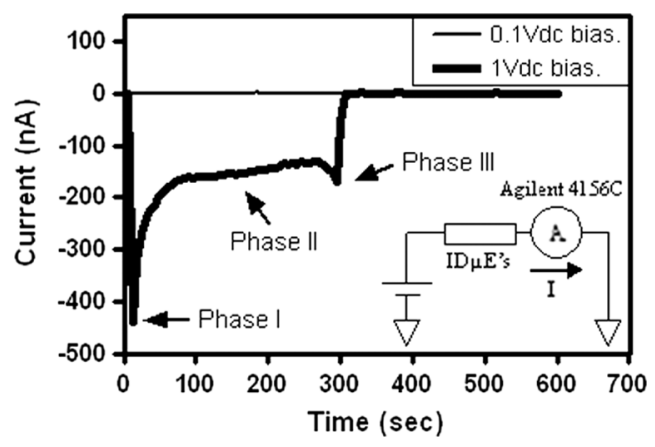

Fig. 7. $I(t)$ curves of the current behavior during drying of $40 \mathrm{~nm}$ gold beads colloidal solution to: (a) $0.1 \mathrm{Vdc}$ bias (thick curve) and (b) $1 \mathrm{Vdc}$ bias (thin curve). The experimental wiring is shown in the inset.

sharply rises when the solution is deposited and reaches negative values in the range of $-400 \mathrm{nA}$, and then decreases towards a "plateau" value in Phase II in the range of $-150 \mathrm{nA}$ in this example. This "plateau" lasts several hundredths of seconds as is the drying phase, while the solution is losing solvent by evaporation to the air. In order to make this phase compatible with the measurement instrumentation, we used the halogen lamp light to accelerate the evaporation process. As the solvent approaches its limit a Phase III is observed where the current shows a clear peak to the range of $-200 \mathrm{nA}$ and then sharply decreases towards zero. 


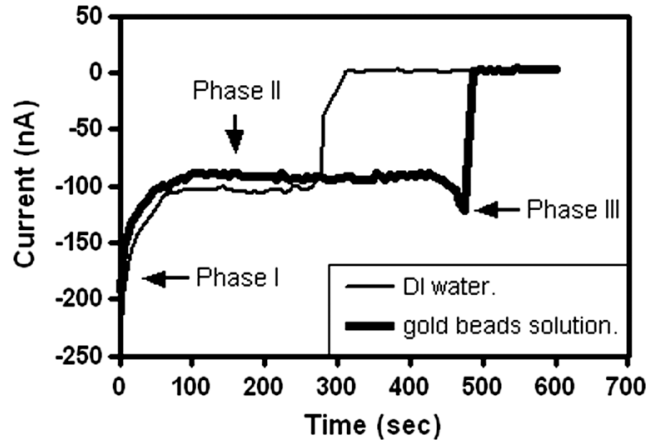

Fig. 8. $I(t)$ curves of the current behavior during drying of: (a) $100 \mathrm{~nm}$ gold beads colloidal solution (thick curve) and (b) DI water (thin curve).

We attributed Phases I and II to the solvent itself and Phase III to the gold nanobeads. This is based on the results shown in Fig. 8 where the $I(t)$ characteristics measured using the gold nanobeads solution is compared with similar transient in which only a droplet of DI water is used instead of the gold solution.

As can be seen the shape of the DI water characteristic does not include the peak of Phase III observed in the gold solution transient. However, Phases I and II are similar exception made of the time scale which is different due to volume and other irrelevant experimental conditions differences.

Phase III is electrically interpreted as the phase where there is little solvent remaining and the gold nanobeads start landing on the substrate, they lose charge as they touch the contacts and then are able to touch each other and aggregate. This explains the current peak at the end of Phase III that only happens for the sample having gold nanobeads in solution. The interpretation of the aggregation is given below. Let us for a moment discuss the relationship that may happen between the steady-state measurements of the previous section with the transient measurements just described. In fact one should expect that the current measured at the end of the transient be equal to the current measured under steady-state conditions for the same bias voltage. In fact this is not the case in our observations, because we have seen a large difference in the current values (in the range of $150 \mathrm{nA}$, in the peak, for the transient results and in the range of $800 \mathrm{nA}$ for the steady-state measurements). The interpretation of these results comes from the different ambient conditions for the two cases. In fact the transient is performed under halogen light and this is precisely used to accelerate the drying process, thereby meaning that the substrate is at a higher temperature than the ambient temperature and hence the sample surface is drier. Compared to that, the steady-state measurements are made under room temperature and humidity conditions (estimated to be in the range of $60 \%-70 \%$ at the time). So the humidity being different helps the conduction of current between the two electrodes, and then not relying only in tunnel conduction. Influence of the heating in the electrical conductance of gold nanoparticle films was studied previously by Prevo et al. [18]. In the same way, they found that conductivity decrease sharply with temperature increase.

Therefore the conductance increase is attributed to two factors: 1) the application of a moderate bias voltage during drying (e.g., $+1 \mathrm{~V}$ ) and 2) the presence of humidity adsorbed on the

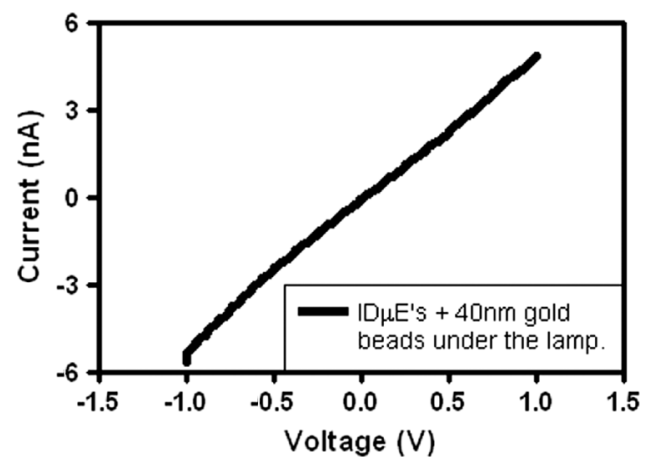

Fig. 9. $I-V$ curve immediately after drying of $40 \mathrm{~nm}$ gold beads colloidal solution by keeping the lamp during the measurement.

sample surface during measurement. Both factors are necessary, but not sufficient one without the other, to obtain a significant current increase.

The $I-V$ curve of gold beads solution after the solution was dried and keeping the halogen lamp can be seen in Fig. 9. In this curve the temperature and humidity conditions are similar to the conditions in which current is measured in the final of the transient state. The influence of the heating in the steady state can be appreciated. As can be seen the value of the electrical current at $+1 \mathrm{~V}$ is around $5 \mathrm{nA}$. This value of current is as low as the values obtained in the final of the transient experiments (Figs. 7 and 8) and in our baseline measurements (Fig. 2) for the same bias voltage because the humidity is not helping the conduction of current when samples are under halogen lamp.

\section{DISCUSSION}

The interpretation of the results described in this work is based on the fact that the gold nanoparticles which are suspended inside the droplet bear a negative charge which prevents them from forming aggregates as they are capable to repel each other. If no electric field is applied during the drying phase, the gold nanobeads distribution on top of the electrodes is totally random and little aggregates are formed. However, when an electric field is applied during the drying process, the coulombian attraction is the most important force which attracts the gold nanoparticles, which are in suspension and negatively charged towards the positive electrode as the solution is drying. This is clearly seen in Fig. 3 where the particles concentrate towards the positive electrode. As the particles start landing and get closer to the electrode plane, they enter a region where the electric field is not uniform as it is maximum in the plane and in between the finger electrodes. This gradient creates a dielectrophoretic attracting the highly conductive beads towards the area of maximum gradient of the electric field (the force is proportional to the gradient of the square of the electric field) that means repelling them from the top of the electrodes and trying to confine them in between the fingers, which is where the electric field is more intense.

As the particles come in contact with the electrodes, the charge of the particles is neutralized by electrons flowing from the metal electrodes and creating an electric current in the external bias circuit (this current correspond to negative charge flowing out of the positive electrode). 
Once the particles are electrically neutral, they tend to aggregate parallel to the electric field lines due to the induced polarization and creating nanosphere chains according to the theory in Jones [19]. In our observations this is combined with the conductive effects of the solvent itself that we have separately identified by performing similar measurements using only DI water.

We can conclude that the application of an electric field of moderate value between interdigitated electrodes, in combination with the humidity on the sample surface, produces significant changes in the conductance between them, both in steady-state and transient conditions. This has been correlated to morphological inspections where the application of this electric field appear to be fundamental to the formation of monolayers and other aggregations of the gold nanobeads which are identified as responsible for the conductivity changes.

The acquired knowledge in gold nanoparticles dynamics from this contribution will be used in the nanoparticles manipulation by means of the selective deposition for biosensor applications. Also this knowledge can be applied to new electronic, quantum or optical devices and to a broad spectrum of new applications.

\section{REFERENCES}

[1] L. Malaquin, C. Vieu, M. Genevieve, Y. Tauran, F. Carcenac, M. L. Pourciel, V. Leberre, and E. Trévisiol, "Nanoelectrode based devices for electrical biodetection in liquid solution," Microelectron. Eng., vol. 73-74, pp. 887-892, Apr. 2004.

[2] J. Spadavecchia, P. Prete, N. Lovergine, L. Tapfer, and R. Rella, “Au nanoparticles prepared by physical method on $\mathrm{Si}$ and sapphire substrates for biosensor applications," J. Phys. Chem. B, vol. 109, no. 37, pp. 17347-17349, Aug. 2005.

[3] Y. Cui, M. T. Björk, J. A. Liddle, C. Sönnichsen, B. Boussert, and A. P. Alivisatos, "Integration of colloidal nanocrystals into lithographycally patterned devices," Nano Lett., vol. 4, pp. 1093-1098, Apr. 2004.

[4] J. T. Sheu, C. C. Chen, P. C. Huang, and M. L. Hsu, "Selective deposition of gold nanoparticles on $\mathrm{SiO}_{2} / \mathrm{Si}$ nanowire," Microelectron. Eng., vol. 78-79, pp. 294-299, Jan. 2005.

[5] S. B. Fuller, E. J. Wilhelm, and J. A. Jacobson, "Ink-jet printed nanoparticle microelectromechanical systems," J. Microelectromech. Syst., vol. 11, no. 1, pp. 54-60, Feb. 2002.

[6] B. Kim, S. L. Tripp, and A. Wei, "Self-organization of large gold nanoparticles arrays," J. Amer. Chem. Soc., vol. 123, pp. 7955-7956, 2001.

[7] M. Kimura, S. Kobayashi, T. Kuroda, K. Hanabusa, and H. Shirai, "Assembly of gold nanoparticles into fibrous aggregates using thiol-terminated gelators," Adv. Mater., vol. 16, pp. 335-338, Feb. 2004.

[8] T. Junno, M. H. Magnusson, S.-B. Carlsson, K. Deppert, J.-O. Malm, L. Montelius, and L. Samuelson, "Single-electron devices via controlled assembly of designed nanoparticles," Microelectron. Eng., vol. 47, pp. 179-183, 1999.

[9] C. Thelander, M. H. Magnusson, K. Deppert, and L. Samuelson, "Gold nanoparticle single-electron transistor with carbon nanotube leads," Appl. Phys. Lett., vol. 79, pp. 2106-2108, 2001.

[10] D. I. Gittins, A. S. Susha, B. Schoeler, and F. Caruso, "Dense nanoparticulate thin films via gold nanoparticle self-assembly," Adv. Mater., vol. 14, pp. 508-512, Apr. 2002.

[11] A. Bezryadin, C. Dekker, and G. Schmid, "Electrostatic trapping of single conducting nanoparticles between nanoelectrodes," Appl. Phys. Lett., vol. 71, pp. 1273-1275, Jul. 1997.

[12] S. I. Khondaker, "Fabrication of nanoscale device using individual colloidal gold nanoparticles," IEE Proc.—Circuits Devices Syst., vol. 151, no. 5, pp. 457-460, Oct. 2004.

[13] M. Giersig and P. Mulvaney, "Formation of ordered two-dimensional gold colloid lattices by electrophoretic deposition," J. Phys. Chem., vol. 97, pp. 6334-6336, Apr. 1993.

[14] M. Giersig and P. Mulvaney, "Preparation of ordered colloid monolayers by electrophoretic deposition," Langmuir, vol. 9, pp. 3408-3413, Jun. 1993.
[15] D. A. Weitz and M. Oliveria, "Fractal structures by kinetic aggregation of aqueous gold colloids," Phys. Rev. Lett., vol. 52, pp. 1433-1436, Apr. 1984.

[16] D. A. Weitz, J. S. Huang, and M. Y. Lin, "Limits of the fractal dimension for irreversible kinetic aggregation of gold colloids," Phys. Rev. Lett., vol. 54, pp. 1416-1419, Apr. 1985.

[17] M. Burghard, G. Philipp, S. Roth, and K. von Klitzing, "Brinding of lateral nanolectrodes with a metal particle chain," Appl. Phys. A, vol. 67, pp. 591-593, Sep. 1998.

[18] B. G. Prevo, J. C. Fuller, III, and O. D. Velev, "Rapid deposition of gold nanoparticle films with controlled thickness and structure by convective assembly," Chem. Mater., vol. 17, pp. 28-35, 2005.

[19] T. B. Jones, Electromechanics of Particles. Cambridge, U.K.: Cambridge Univ. Press, 1995.

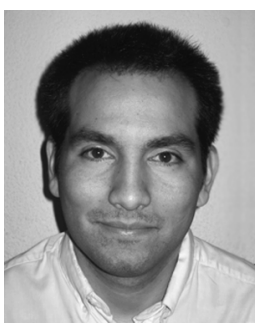

Enrique Valera was born in Lima, Peru in 1977. He received the B.S. degree in electronic engineering from the Catholic University of Peru in 2003. He is currently working toward the Ph.D. degree in the Micro and Nanotechnologies group of Technical University of Catalonia, Spain.

Since 2002 he is working in biosensors, silicon micromachining nanodevices, and porous silicon technologies for sensors development. His main research areas are now biosensors, nanotechnologies, and MEMS.

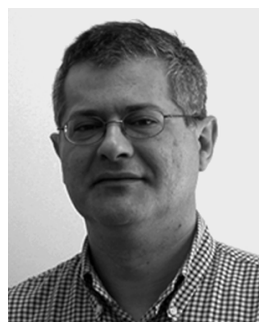

Ángel Rodríguez (M'96) graduated in telecommunication engineering from the Universidad Politécnica de Cataluña, Spain.

From 1987 to 1992 he worked at IMEC, Belgium, toward his doctoral degree in the field of polysilicon thin-film transistors. In 1993, he became an Associate Professor in the Escuela Técnica Superior de Ingenieros de Telecomunicación de Barcelona, Spain. He has worked in solar cells, bipolar transistors, polysilicon thin-film transistors, and MEMS. Concerning MEMS he has worked in flow sensors, accelerators, different kinds of artificial noses based in metallicoxides or polymers resonating structures, RF MEMS, MEMS actuators, porous silicon-based MEMS/MOEMS, and more recently in bio-MEMS.

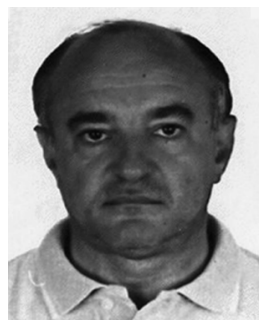

Luis M. Castañer (SM'92) is a Professor with the Departamento de Ingeniería Electrónica de la Escuela Técnica Superior de Ingenieros de Telecomunicación at the Universidad Politécnica de Cataluña, where he received his Doctor Ingeniero de Telecomunicación, following his undergraduate studies (Ingeniero Superior de Telecomunicación. from the Universidad Politécnica de Madrid, Spain, in 1971 and Diplóme d'Etudes Approfondies (D.E.A.) en Physique Spatiale and Docteur-Ingenieur by the Université Paul Sabatier in Toulouse, France, in 1973). He has also been Dean of E.T.S.I. Telecomunicación and Head of Departamento de Ingeniería Electrónica, and has held several positions in Research Agencies and Committees: representative in the Comite de Gestion y Coordinacion on non nuclear energies, DGXII Comission of the EU, Head of the Programa Nacional de Tecnologías de la Información y Comunicaciones of the CICYT (1992-1994), and Coordinator of the Technology Foresight at the Agencia Nacional de Evaluación y Prospectiva. He has contributed to semiconductor device research, covering solar cells in various aspects: technology of CIS, space degradation of Silicon and GaAs devices, and has contributed to the theory and technology of advanced bipolar transistors with polysilicon emitters and its application to high-efficiency silicon solar cells, in particular, to the emitter resistance of these devices. He has also contributed to the design, simulation, and monitoring of photovoltaic power plants and systems, and is active in the microsystems technology area, working on flow sensors, power devices for MEMS, and electrostatic actuators. He is a Member of the Association and Charter of Telecommunication Engineers in Spain, and a Member of the Spanish Engineering Academy. 